\title{
Systemic Obstacles to Addressing Research Misconduct in Higher Education: A Case Study
}

\author{
James Golden ${ }^{1}$ (D) $\cdot$ Catherine M. Mazzotta ${ }^{1}$ (D) Kimberly Zittel-Barr $^{1}$ (D)
}

Accepted: 19 August 2021 / Published online: 29 August 2021

(C) The Author(s), under exclusive licence to Springer Nature B.V. 2021

\begin{abstract}
Several widely publicized incidents of academic research misconduct, combined with the politicization of the role of science in public health and policy discourse (e.g., COVID, immunizations) threaten to undermine faith in the integrity of empirical research. Researchers often maintain that peer-review and study replication allow the field to selfpolice and self-correct; however, stark disparities between official reports of academic research misconduct and self-reports of academic researchers, specifically with regard to data fabrication, belie this argument. Further, systemic imperatives in academic settings often incentivize institutional responses that focus on minimizing reputational harm rather than the impact of fabricated data on the integrity of extant and future research.
\end{abstract}

Keywords Research misconduct · Research fraud · Data falsification · Fals-Stewart · Behavioral couples therapy $\cdot$ Retracted publications $\cdot$ Higher education $\cdot$ Institutional responsibility

A case study of the work of Dr. William Fals-Stewart, a prolific and widely-respected researcher of Behavioral Couples Therapy (BCT), who faced serious allegations of research misconduct prior to his death, including data falsification, will be presented to illustrate the insidious consequences of fraudulent data on an entire field of research. Additionally, the role of academic institutions, co-authors, and journals will be explored to identify opportunities to mitigate the harm caused by research misconduct.

In the mid-1970s, $72 \%$ of Americans trusted the mainstream print and television media to report the news fully, accurately, and fairly; now, however, only $47 \%$ surveyed indicate a similar level of trust, hitting a low of 32\% in 2016 (Brenan, 2020). Concurrent with this loss of trust in mainstream news sources has been a similar erosion of faith among lay persons in the accuracy and impartiality of the scientific community (Leiserowitz et al., 2013). While some of this skepticism can be attributed to recent political rancor regarding the innumerable missteps at the federal and state level in providing evidence-based guidance during the COVID-19 pandemic, this increasing mistrust has been building since the late

James Golden

goldenjc@buffalostate.edu

1 Social Work Department, Buffalo State College SUNY, 1300 Elmwood Ave, Buffalo, NY 14222, USA 
1990s due to several widely-publicized incidents across disciplines in both the natural and social sciences involving the publication of studies based on dubious methods and/or falsified data (Wakefield et al., 1999; Stapel \& Lindenberg, 2011; Carpenter, 2012).

For some in the research community, these incidents of research misconduct, in which articles were retracted and the perpetrators sanctioned for their actions, underscore a longheld belief that scientific inquiry is inherently "self-correcting" (Crocker \& Cooper, 2011; Kraut, 2011), thus rendering concerns about the integrity of the research process unnecessary. Conversely, others argue that the actions taken to address these acts of misconduct are an anomaly given the prevalence of research misconduct, and that the investigatory process moves at a glacial pace, thus allowing publications based on falsified data to become embedded within a given field's knowledge base for years before being formally retracted, if at all (Ioannidis, 2012; Cofnas, 2016).

Estimates of the prevalence of data falsification vary greatly. For example, though audits of federally sponsored clinical trials find that research misconduct is exceedingly rare $(0.01 \%)$, the rate increases exponentially when researchers are asked if they have ever falsified data (2\%) (Finelli, 2009) or have first-hand knowledge of a colleague having falsified data (17\%) (Sheehan et al., 2005). Article retractions represent another indicator of the prevalence of academic research misconduct. From 2000-2014, the number of article retractions increased ten-fold, as did the number of journals issuing retractions (Brainard \& Yao, 2018), and a review of retracted articles in the biomedical journal database, PubMed, found that $43 \%$ of the retractions were attributable to fraud or falsification of data (Fang et al., 2011). Regardless of the field of study, it is generally understood that the actual rate of research misconduct, and specifically, the falsification of data, is vastly underreported (Lee, 2012).

The lack of accuracy in assessing the breadth of academic research misconduct has impeded researchers' ability to improve understanding of the impetus for engaging in such acts. Consequently, the problem of academic research misconduct is often presented as largely attributable to the pressures of the academic rank and tenure process, which emphasizes publication productivity for continued employment, along with ongoing pressures that full-time tenured and tenure-track faculty face, such as securing external funding and publishing in the most prestigious journals in one's field of study (Holtfreter, 2019). Though these factors clearly contribute to the problem of academic research misconduct, the tendency to contextualize research misconduct at the individual level in terms of coping skills, workplace culture, and personal ambition fails to recognize the larger institutional and systemic forces that unintentionally incentivize misconduct.

The following case study will present a pattern of research misconduct by Dr. William Fals-Stewart (NYS AG, 2010), a prominent researcher in the field of intimate partner violence (IPV), that took place over the course of nine years, during which he was employed at two separate R1 universities located in Western New York. It is important to note at the outset that, although credible accusations were first made in 2004 regarding suspected research misconduct, to date, Dr. Fals-Stewart's 155 research publications have been cited more than 11,400 times, and zero articles have been retracted.

\section{Case Study}

\section{Behavioral Couples Therapy}

Behavioral Couples Therapy (BCT) is a behavior-based treatment model developed in the late 1960s and is predicated upon the belief that interactions within a drug- or 
alcohol-addicted client's intimate interpersonal relationship can help or hinder the recovery process. Consequently, practitioners utilizing BCT with their substance abusing clients actively seek to involve their clients' significant others in treatment by having them attend weekly counseling sessions, and by participating in homework tasks during the intervening weeks, to reduce relationship dysfunction that may interfere with recovery.

Empirical evidence of BCT's effectiveness in improving recovery outcomes and relationship functioning led researchers to examine tertiary treatment effects, and by the early late 1990s, a growing body of research assessing BCT's impact on intimate partner violence (IPV) among substance-abusing couples began to emerge, but not without controversy. Specifically, advocates for victims of IPV have long-argued that couples counseling with IPV falsely implies that the victim shares responsibility for causing and ending their partner's violence, while also cautioning that the treatment modality increases the potential for further acts of violence by the perpetrator resulting from in-session conflict between the partners and/or perceived challenges to the perpetrator's authority if the victim speaks freely and honestly about the perpetrator's actions (Karakurt et al., 2016; Tomisich et al., 2015).

\section{Dr. William Fals-Stewart}

Nonetheless, research on BCT with IPV couples proceeded in earnest, and by the mid2000s, William Fals-Stewart was considered one of the foremost experts in BCT treatment (Hazelden, 2008). In 2000, having previously held faculty positions at Harvard and Old Dominion Universities, he moved to the University of Buffalo's Research Institute on Addictions (UBRIA), where he was the principal investigator (PI) or co-principal investigator (Co-PI) on 7 federally funded research grants concurrently, totaling approximately $\$ 12$ million, before leaving under a cloud of suspicion in 2005 due to allegations of misconduct related to his research activities (Herbeck, 2010).

After a brief stint at the Research Park Triangle in North Carolina, he landed at the University of Rochester's School of Nursing, Addiction, and Family Research in 2007, only to resign in 2009, having failed to receive tenure. Despite the chaos surrounding his employment status from 2000-2009, Fals-Stewart was a prolific and widely respected researcher in the field of BCT, and developed a comprehensive portfolio of publications, including a growing body of research and treatment manuals regarding the use of BCT with IPV couples. By the time his research career ended, he had amassed more than 150 peer-reviewed publications, served as the convention chair for the American Psychological Association's 2003 national convention, co-authored the book, Behavioral Couples Therapy for Alcoholism and Drug Abuse (2006), which was subsequently listed on the now-defunct Substance Abuse and Mental Health Services Association's (SAMHSA) National Registry of Evidence-Based Programs and Practices, and received the 2007 Hazelden Foundation's Dan Anderson Research Award. The latter award honors one published research study annually for its contribution to advancing empirical research in the field of addictions and recovery. Past recipients include faculty from Harvard University and Yale University.

\section{Red Flags}

From approximately 2000-2005, while at UBRIA in Buffalo, NY, Fals-Stewart became a widely respected researcher in BCT. As noted, Fals-Stewart's time at UBRIA was remarkable for his prodigious research and publication productivity, along with managing nearly 
$\$ 12$ million in federally funded grants through the National Institute for Drug Abuse (NIDA). Nonetheless, several peculiarities manifested in the way he conducted himself and his research. Most notably, according to the grant proposals submitted to NIDA, meeting the aims of his projects would require recruiting more than 900 research participants. However, despite conducting these projects in Erie County, NY, home to nearly one million residents, recruitment was anemic at best, numbering in the single digits most months, and wholly inconsistent with the robust data collection activity described by Fals-Stewart in his publications, annual reports, and grant writing activities (NYSAG, 2010). In fact, during the two years that the first author of this manuscript served as Project Director on two of Fals-Stewart's NIDA grants, the research team at UBRIA frequently went weeks without enrolling new research subjects.

Project staff often worried about the lack of new subject recruitment, which was particularly noticeable relative to comparable projects running concurrently at UBRIA. FalsStewart dismissed such concerns, insisting that the vast majority of the research subjects for his studies were recruited through a second data collection site called "Alpha Foundation," which was located three hours away, in a county with a population $1 / 10^{\text {th }}$ the size of Erie. Despite working under the same grant, and using the same informed consent, data collection procedures, and treatment conditions, the staff from UBRIA and Alpha Foundation were not allowed to interact in person, or by phone or email.

Further, Fals-Stewart's relationship with the "Alpha Foundation" remains unclear, including the foundation's name. Throughout Fals-Stewart's career, the foundation was listed among his acknowledgements on his publications as "Alpha Addictions Research, Ithaca, NY" (Fals-Stewart \& Lucente, 1994) or "Alpha Foundation" (Fals-Stewart et al., 2003). Irrespective of the name change, public filings and information regarding either titled entity is lacking, and it appears Fals-Stewart was the sole researcher receiving financial support from said foundation.

Lastly, perhaps owing to the lack of activity on his projects at UBRIA, or to a rather brazen effort to give the appearance of robust data collection, on more than one occasion, Fals-Stewart had his research assistants "mockup" dozens of completed research subject folders for "practice." It was unclear what "practice" purpose these served, as they were not used to assist with training research or clinical staff, and in fact, were simply stored in Fals-Stewart's office.

\section{Allegations of Research Misconduct}

In 2003, while working to address a review panel's request for additional information regarding data collection and analysis of a manuscript based on data from and co-authored with Fals-Stewart, this article's first author made repeated requests to Fals-Stewart for access to the relevant data files, to no avail. Subsequently, with no warning and for reasons never made clear, Fals-Stewart restricted all access by this author to all project related documents. Further, Fals-Stewart deleted the manuscript noted above, which had been accepted pending revisions, and withdrew his mentorship support from this author's nearcompleted K01 award application.

Given the bizarre circumstances that transpired surrounding the manuscript revisions, which added to already existing concerns regarding Fals-Stewart's conduct, this author reached out to the IRB at the University of Buffalo (UB), which was responsible for overseeing UBRIA's research activities. The secretive nature of Fals-Stewart's behavior, combined with a volatile temper, kept his research team at arm's length, making it difficult to 
offer substantive documented evidence of misconduct to the IRB. Nonetheless, in addition to disclosing the events that had transpired regarding the manuscript, the IRB was informed of concerns regarding potential discrepancies between actual and reported subject recruitment totals, along with Fals-Stewart's practice of having project staff create fake subject folders. Finally, this writer informed the IRB that on several occasions, Fals-Stewart issued directives to project staff that were not consistent with the protocols approved by the IRB, including recruitment criteria and treatment conditions.

By 2005, Fals-Stewart was forced out of UBRIA due to new allegations of data falsification levied in September 2004 (Herbeck, 2010). In 2007, he was the subject of an investigation by the State University of New York (SUNY) for falsifying data during his time at UBRIA. During that investigation, SUNY investigators sought to interview three of Fals-Stewart's current and former project staff members, but Fals-Stewart claimed that they were busy and unavailable for in-person interviews. The investigators agreed to conduct the interviews by phone. Based on the testimony of these project staff, Fals-Stewart was cleared of any wrong-doing by SUNY in 2008.

\section{NYS Investigation}

Seemingly out from beneath a cloud of suspicion and having landed on his feet as research faculty at the University of Rochester, Fals-Stewart, chose to sue SUNY for \$4 million for defamation of character because of the investigation. Given that SUNY is a state agency, the New York State Attorney General's (NYSAG) office was tasked with defending SUNY against Fals-Stewart's lawsuit. Wielding expansive resources and a broader investigative mandate to defend its client, the NYSAG's office soon discovered that Fals-Stewart's defense against the original accusations of falsifying data, was in fact predicated on falsified testimony (NYSAG, 2010).

While preparing its defense, the NYSAG's office interviewed the three staff members who testified on Fals-Stewart's behalf during the 2005 investigation and learned that they were actors, paid by Fals-Stewart, who had provided them with scripted testimony, to participate in what he described as a "mock trial." As a result, Fals-Stewart was charged with multiple felonies, including grand larceny, perjury, identity theft, offering a false instrument and falsifying business records (NYSAG, 2010). One week after the arrest, FalsStewart died in his home at 48 years of age, of unknown causes, leaving behind a wife and two young children (Warner, 2010).

\section{Discussion}

Though Fals-Stewart bears sole responsibility for his transgressions, his actions did not take place in isolation. Rather, Fals-Stewart's actions represent the confluence of systemic factors that prevail upon those in higher education institutions, particularly junior, tenuretrack faculty and those in "soft" money positions. While the rank and tenure process in academia is often singled out for its role in increasing the likelihood of ethical lapses, that process serves the same performance evaluation function that exists in many other nonacademic professions that are similarly hierarchical and highly competitive. Thus, the pressures created by the rank and tenure process are not unique to academia.

However, what is unique is that responsibility for oversight of the key performance indicator for rank and tenure, publications, is left to a network of loosely affiliated journals that 
are not privy to inner-workings of a given author's research laboratory. Consequently, the publication process can only reasonably rely on the assumption that articles are submitted in good faith, and that the peer review and replication processes will root out suspect research. However, the U.S. Office of Research Integrity (ORI) conservatively estimated the rate of research misconduct to be $60 \times$ more than the number of cases opened by ORI annually (Gallup, 2008). Thus, colleges and universities have essentially abdicated responsibility for ensuring the integrity of the research productivity of their respective faculty to a publication process that was not intended to serve as the gate keeper for the rank and tenure process, especially as concerns abound from within the research community regarding the veracity of the fundamental assumption that scientific inquiry is inherently self-correcting. To this end, it is imperative to begin to explore strategies to address the most salient systemic obstacles that confound efforts to root out research misconduct.

\section{Institutional-Responsibility}

Culpability for academic research misconduct is far more complex than often portrayed. Principal investigators bear primary responsibility for maintaining fidelity to their respective study protocols and abiding by established research ethics and are thus responsible for actions that breach these obligations. However, given the financial benefit for administering grant funding, termed an "indirect cost," along with the contribution of each grant award towards an institution's Carnegie classification, and the desire to avoid negative media attention, academic institutions often succumb to such pressures by working to protect their respective organizations at the expense of the integrity of scientific inquiry (Titus et al., 2008). Institutions are particularly vulnerable to the potential for inadequately addressing research misconduct when a researcher such as Fals-Stewart contributes disproportionately to the institution's overall funding and publication productivity. From 2003-2004, of the 33 federally funded projects, totaling approximately $\$ 45$ million awarded to UBRIA's 22 principal investigators, Fals-Stewart was the PI or Co-PI on $21 \%$ (7) of the projects, totaling \$12 million, or approximately $27 \%$ of UBRIA's total funding during that fiscal year.

This is not to imply that institutions do not intervene, but institutional imperatives often emphasize minimizing and/or quietly moving past allegations or findings, potentially resulting in perfunctory or performative investigations and disciplinary actions. This, in turn, works against the goal of minimizing damage caused by fraudulent studies infecting the extant literature and knowledge base. It is worth noting that Fals-Stewart left UBRIA in 2005 due to allegations of research misconduct but held research positions at the Research Triangle Institute (RTI) in North Carolina, and then University of Rochester (UR) School of Nursing, until a few months before his death in 2010. A search for Fals-Stewart's name on the RTI website yields three results, and the University of Rochester's site yields four, but a search of UBRIA's website, where the allegations originated, finds none.

Fals-Stewart's exit from UBRIA illustrates the competing interests within academic institutions when they commodify and prioritize research productivity for the purposes of tenure decisions and national rankings (Csiszar, 2020). Ultimately, it is important to note that Fals-Stewart's downfall was not brought about because of UBRIA's oversight of his research activity, or the actions they took to protect the integrity of research science and the general public from Fals-Stewart's misconduct. In fact, despite ongoing concerns and subsequent investigations regarding data falsification on federally funded grants that hung over Fals-Stewart for nearly 4 years, his quiet departure from UBRIA allowed him to continue to ply his craft elsewhere, earning a national award for his research and landing a faculty 
position at another R1 university while he was concurrently being investigated by SUNY. Though SUNY concluded in 2008 that there was insufficient evidence to warrant disciplinary action (Herbeck, 2010), Fals-Stewart would end up resigning from UR in 2009, having been denied tenure (Campus Times, 2010).

Unfortunately, the decision by UBRIA and UR to address Fals-Stewart's actions as they did is not unique. DuBois et al. (2013) surveyed 194 research integrity officers from universities in the United States and found that $88 \%$ reported having conducted investigations in the prior two years, with more than $50 \%$ of those investigations triggered by credible accusations of research misconduct. However, federal guidelines do not require universities to report the results of these investigations. Instead, despite more than two-thirds of whistleblowers reporting at least one act of potential retribution for their actions, and nearly half reported being pressured to withdraw the allegations (Titus et al., 2008), ORI relies on institutions to investigate and take disciplinary action when deemed necessary.

Consequently, the responsibility that academic institutions owe to the field of science to establish and maintain a culture of integrity and accountability is too often sacrificed in favor of remedies that prioritize revenue and reputations. However, in the absence of a legal imperative to publicly disclose confirmed instances of research misconduct and take specific, substantive action to mitigate the dissemination of fraudulent data, there are an unknown number of researchers actively publishing in their field after having been allowed to quietly resign and take their grant money elsewhere, just as Fals-Stewart did at least twice.

Thus, it imperils the field of research to allow academic institutions to continue framing their role in scientific inquiry as that of neutral observers. Rather, academic institutions have repeatedly demonstrated that their interests, intentional or not, can often run counter to the needs of the scientific community. Compounding this dynamic is ORI's choice to defer responsibility to colleges and universities for the investigation and adjudication of allegations of research misconduct. Consequently, higher education has established a research culture in which colleges and universities can reap significant financial and reputational rewards from a tenure process that compels feverish grant and publication activity, only to accept little to no responsibility for overseeing the actions of faculty in order to satisfy the conditions for continued employment.

As with any corrective action, the incentives for establishing and maintaining ethical standards must outweigh the benefits of acting otherwise. For many colleges and universities, the fear of negative public relations consequences that might result from calling attention to research misconduct have the potential for creating a greater imperative to ignore, stifle, or minimize acts of research misconduct than to report them and ensure that steps are taken to protect the integrity of the given researcher's field of study. To this end, though the responsibility for fiscal oversight that institutions assume when administering grant funding is narrowly defined as ensuring the appropriate use and accurate tracking of grant expenditures, it is reasonable to extend that definition to include ensuring that the funding was not used in the support of fraudulent research activities.

Doing so would require that colleges and universities be compelled to realign institutional priorities from a traditional focus on grant procurement and management, to an organizational ethos that embraces its role in protecting the integrity of scientific inquiry. This is particularly important given that research productivity has become the principal mechanism by which higher educational institutions evaluate their faculty and distinguish themselves from peer institutions (Gingras, 2020).

Specifically, in cases in which fraudulent behavior is confirmed, the institution should bear responsibility for not only reimbursing the funding source for the grant and indirect funding 
received, but also risk a formal sanction if it is found that the college or university did not make a good faith effort to address concerns or allegations of fraud. For example, UB never followed up on the concerns this writer voiced to the IRB in 2003 regarding data collection on Fals-Stewart's projects. Additionally, despite having been one of Fals-Stewart's longestserving project directors during his time at UB, this writer was not contacted as part of the 2004 or 2007 investigations.

Sanctions could include informing the institution's accrediting body of the infractions, placing the college or university on probation requiring future grant applications to include additional procedures for monitoring grant activities, and ultimately barring the college or university from receiving federal funding for a period of time.

\section{Co-Authors}

The foremost risk in allowing fraudulent data is to the populations for whom treatment and policy decisions are informed by such data. Given that Fals-Stewart was one of the most prolific researchers in the area of BCT and his work was, and to some extent still is, ubiquitous among articles on the subject of BCT, his influence on that field of research is vast and incalculable. With such a breadth and depth of influence, it is unknown whether empirical support for BCT with such couples would remain as robust in the absence of Fals-Stewart's fraudulent contribution to the research. IPV carries a serious potential risks to victims and it is imperative that evidence for interventions be empirically sound. BCT methods were not considered appropriate for this population prior to Fals-Stewart's research; however, his research played a significant role in establishing BCT as both effective and safe. Following discovery of data falsification, almost nothing has been done to draw attention to the consequences of Fals-Stewart's actions, or to re-assess the accuracy of his findings. In fact, FalsStewart's research has been cited more than 7,000 times since his death, which was 5 years after he left UB due to allegations of fraud.

As it stands, Fals-Stewart is dead. His actions while at UBRIA, RTI, and UR have largely been exorcised from their websites, and the author information page for the book that FalsStewart co-authored on the Guilford Press website, Behavioral Couples Therapy for Alcoholism and Drug Abuse (2006), only notes that Fals-Stewart died in 2010. Thus, any attempt at due diligence based on information provided by his former institutions and publisher would give no indication that there is reason to question the validity of his research.

Consequently, only those who worked with him or were privy to the investigations are aware of what took place, but that is no small number. Fals-Stewart worked with more than 50 co-authors, from academic institutions throughout the United States, and, to date, it does not appear that any of them have sought to have their publications with Fals-Stewart retracted. Consistent with the barriers presented heretofore, the pressures of academic publishing and grant procurement create significant disincentives for co-authors to seek retractions.

Specifically, a faculty member's publishing record is used to determine continued employment during the tenure process, as well as serving as the basis for grant applications. As such, the secondary and tertiary consequences for a co-author to come forward, especially tenure-track faculty, are foreboding. For example, Mongeon and Larivière (2016) found that co-authors of articles retracted due to fraud experienced a significant decline in publication productivity in the subsequent years and, $28 \%$ of co-authors who were innocent of wrongdoing did not publish another article in the five years following the retraction. Consequently, given that publication productivity is the primary metric used in 
determining tenure decisions, an article retraction is potentially career-ending for junior faculty, even among those who are innocent of wrongdoing.

Fals-Stewart worked with several early-career researchers, many of whom co-authored several articles with him before pursuing their own grant funding and research careers. These co-authors began building their research portfolios, citing their own work with FalsStewart, along with his many other publications, otherwise unaware that their findings may have been the byproduct of research fraud. The net result is that, not only did Fals-Stewart's falsified data gain ever greater credibility as it continued to be cited by a wider array of researchers across multiple institutions, but also, the sheer volume of citations allowed his research to propagate quickly and become embedded within the extant literature and knowledge base of BCT.

Responsibility for addressing the ongoing dissemination of fraudulent research findings must begin at the institution level, foremost by allowing co-authors to come forward when they are privy to information that calls into question the legitimacy of an article they have co-authored. That, however, is the easy part. The difficulty is in establishing policies that encourage, or at least do not punish, the ethically correct action of removing articles derived from questionable research methods from one's curriculum vitae. For example, policies might allow un-tenured faculty to extend their "tenure clock," which would grant them extra time to rebuild their publication record before they are evaluated for rank and tenure. Alternatively, assuming the junior faculty member, as co-author, contributed to the publication during the writing stages, rather than during the period in which data falsification took place, their contribution in terms of authorship leading to a publication would remain recognized towards publication requirements for tenure. However, regardless of the manner in which academic institutions work with junior faculty to remediate gaps in tenure progress caused by the loss of retracted articles, it is imperative, in all cases and at any rank, retracted publications must remain on one's vitae.

Notably, irrespective of whether a researcher is applying for external funding or simply posting their publication record on their institutional faculty biographical webpage, it should become standard practice that all publications, including those retracted, must be included on all official records of publications. While it is understood that there is a great deal of stigma associated with a retraction, as misconduct is often assumed, academic research, and researchers themselves, must be transparent. Further, requiring that retracted publications be properly documented begins the process of normalizing ethical decision making in research publication practices. Simply put, allowing authors of retracted articles to expunge them from their official professional documentation so as to avoid the embarrassment of having to disclose such an incident undermines the integrity of academic research for the sake of protecting a given researcher's reputation.

\section{Meta-Analyses and Systematic Reviews}

The persistence of Fals-Stewart's impact on BCT research cannot be overstated, as his work has often been featured, sometimes disproportionately, in meta-analyses and systematic reviews of couples treatments for IPV and with other populations. For example, Karakurt et al.'s (2016) systematic review of six published clinical trials of BCT and IPV, three of which were authored by Fals-Stewart, found significant reductions in IPV compared to traditional IPV treatment modalities or no treatment control groups. Karakurt concluded, "there is certainly reason to reevaluate the role of couples therapy in IPV treatment and cautiously increase its application (p. 578)." Likewise, Fals-Stewart's 2009 study of 
BCT with same sex couples was included in McGeough's (2020) systematic review, which found continued support for the efficacy of BCT in substance abuse settings. Fals-Stewart's study was one of two that were singled out for having "employed the strongest methods (p. 205)." Lastly, despite noting "awareness" of the arrest and allegations of fraud against FalsStewart in the Discussion section of their scoping review of family-based substance abuse interventions, Cassidy and Poon (2019) cited his work 55 times and highlighted BCT as having "the largest base of evidentiary support (p. 357)."

As such, consistent with the Committee on Publication Ethics' (COPE) 2019 Retraction Guidelines, once a retraction has been issued, the authors of any systemic reviews or meta-analyses that included the retracted article in their analyses must be notified and encouraged to correct or retract their publication accordingly. While onerous, this is a necessary step, as a retraction only focuses on the article in question, otherwise ignoring the cumulative impact that the article has had on a given field of study. For example, Powers et al's (2008) meta-analysis of BCT for substance abuse, in which six of the twelve studies included were authored or co-authored by Fals-Stewart, concluded that there was "a clear overall advantage of including BCT compared to individual-based treatments (p.952)." This meta-analysis has since been cited more than 350 times.

Wray et al. (2016), included the following note in the Methods section of their systematic review of substance abuse interventions with male, same-sex couples, when noting selection criteria: "An additional study by FalsStewart (sic), O'Farrell and Lam (Journal of Substance Abuse Treatment. 2009 Dec; 37(4): 379-387) met the criteria for inclusion in the review but was excluded based on advice from the editor-in-chief of the Journal of Substance Abuse Treatment that the journal has a general policy of advising authors to exclude the study from reviews because of concerns about the study's validity (p. 150)." Despite such a warning, Fals-Stewart et al. (2009) has not been retracted from Journal of Substance Abuse Treatment, and has been cited nearly 90 times since its publication, including the McGeough (2020) systematic review noted above. Additionally, none of Fals-Stewart's other 10 articles published in the Journal of Substance Abuse Treatment have been retracted, or otherwise flagged as necessitating the type of caution offered Wray et al., either.

\section{Conclusion}

Academic research is a high-pressure environment that unintentionally incentivizes research misconduct and disincentivizes transparency in response to incidences of academic misconduct. The prevalence of misconduct, specifically the falsification of data, not only runs counter to sound empirical practices and fundamental research ethics but has the potential to undermine decades of research dedicated to identifying safe and effective interventions for vulnerable populations. Further, institutional and systemic policies and accepted patterns of behavior that allow such misconduct to go undetected, unreported, or ignored, run the risk of calling into question the field's ability to self-police, self-correct, and, ultimately, maintain the public trust in scientific objectivity and integrity. Efforts must be made to ameliorate the systemic impediments to effecting long-term, meaningful policy changes to discourage research misconduct.

Acknowledgements The authors wish to thank Drs. Robin Pugh Yi and Ramona Santa Maria for their assistance in preparing this manuscript. 


\section{Declarations}

Conflict of Interest We have no known conflicts of interest to disclose.

\section{References}

Arrested ex-professor dies in home. (2010, February 25). Campus Times. Retrieved from https://www. campustimes.org/2010/02/25/arrested-ex-professor-dies-in-home/

Brainard, J. (2018). What a massive database of retracted papers reveals about science publishing's 'death penalty'. Science. Retrieved from https://www.sciencemag.org/news/2018/10/what-massive-databaseretracted-papers-reveals-about-science-publishing-s-death-penalty

Brenan, M. (2020). Americans remain distrustful of mass media. Gallup News Service, N.PAG.

Cofnas, N. (2016). Science Is not always "self-correcting". Foundations of Science, 21, 477-492.

Carpenter, S. (2012). Harvard psychology researcher committed fraud, US investigation concludes. Science. Retrieved from https://www.sciencemag.org/news/2012/09/harvard-psychology-researcher-committedfraud-us-investigation-concludes

Cassidy, A., \& Poon, A. W. C. (2019). A scoping review of family-based interventions in drug and alcohol services: Implications for social work practice. Journal of Social Work Practice in the Addictions, 19, 345-367.

Committee on Publication Ethics. (2019). Retraction guidelines. COPE Guidelines. Retrieved from https:// publicationethics.org/files/cope-retraction-guidelines-v2.pdf

Crocker, J., \& Cooper L. (2011). Editorial: Addressing scientific fraud. Science, 334, 1182. https://doi.org/ 10.1126/science.1216775. Retrieved from https://www.sciencemag.org.proxy.library.uu.nl/content/ 334/6060/1182.full

Csiszar, A. (2020). Gaming metrics before the game: Citation and the bureaucratic virtuoso. In M. Biagiolo and A. Lippman (Eds.) In Gaming the metrics: Misconduct and manipulation in academic research (pp. 31-42). MIT Press. https://doi.org/10.7551/mitpress/11087.003.0003

DuBois, J. M., Anderson, E. E., \& Chibnall, J. (2013). Assessing the need for a research ethics remediation program. Clinical and Translational Science, 6, 209-213.

Fals-Stewart, W., \& Lucente, S. (1994). The effect of cognitive rehabilitation on the neuropsychological status of patients in drug abuse treatment who display neurocognitive impairment. Rehabilitation Psychology, 39, 75-94. https://doi.org/10.1037/h0080316

Fals-Stewart, W., \& O'Farrell, T. J. (2003). Behavioral family counseling and naltrexone for male opioiddependent patients. Journal of Consulting and Clinical Psychology, 71, 432-442. https://doi.org/10. 1037/0022-006X.71.3.432

Fals-Stewart, W., O'Farrell, T. J., \& Lam, W. K. (2009). Behavioral couple therapy for gay and lesbian couples with alcohol use disorders. Journal of Substance Abuse Treatment, 37, 379-387. https://doi.org/10.1016/j. jsat.2009.05.001

Fanelli, D. (2009). How many scientists fabricate and falsify research? A systematic review and meta-analysis of survey data. PLoS One, 4, 1-11.

Fang, F. C., \& Casadevall, A. (2011). Retracted science and the retraction index. Infection and Immunity, 79, 3855-3859.

Gallup Organization for Office of Research Integrity. (2008). Final Report: Observing and Reporting Suspected Misconduct in Biomedical Research.

Gingras, Y. (2020). The transformation of the scientific paper: From knowledge to accounting unit. In M. Biagiolo and A. Lippman (Eds.) In Gaming the metrics: Misconduct and manipulation in academic research (pp. 43-55). MIT Press. https://doi.org/10.7551/mitpress/11087.003.0004

Hazelden. (2008). Fals-Stewart earns 2007 Dan Anderson Research Award. Voice, 13, 13. Retrieved from https://www.hazelden.org/web/public/document/voice_win08.pdf

Herbeck, D. (2010). Researcher accused of using actors at hearing testimony at UB led to misconduct acquittal. Retrieved from https://buffalonews.com/news/researcher-accused-of-using-actors-at-hearingtestimony-at-ub-led-to-misconduct-acquittal/article_4a734f44-5deb-5ca6-ab1e-f23c0f9ff871.html

Holtfreter, K., Reisig, M.S., Pratt, T.C., \& Mays, R.D. (2020) The perceived causes of research misconduct among faculty members in the natural social and applied sciences. Studies in Higher Education, 45, 2162-2174. https://doi.org/10.1080/03075079.2019.1593352

Ioannidis, J. P. (2012). Why science is not necessarily self-correcting. Perspectives in Psychological Science, 7, 645-654. 
Karakurt, G., Whiting, K., van Esch, C., Bolen, S. D., \& Calabrese, J. R. (2016). Couples therapy for intimate partner violence: A systematic review and meta-analysis. Journal of Marital \& Family Therapy, 42, 567-583.

Kraut, A. G. (2011). Despite occasional scandals, science can police itself. Chronicle of Higher Education. Retrieved from https://www.chronicle.com/article/despite-occasional-scandals-science-can-police-itself/

Lee, J. (2012). The past, present, and future of scientific misconduct research: What has been Done? What needs to be done? Journal of the Professoriate, 6, 67-83.

Leiserowitz, A. A., Maibach, E. W., Roser-Renouf, C., Smith, N., \& Dawson, E. (2013). Climategate, public opinion, and the loss of trust. American Behavioral Scientist, 57, 818-837.

McGeough, B., \& Aguilera, A. (2020). Clinical interventions with sexual minority clients: Review, critique, and future directions. Journal of Gay \& Lesbian Social Services, 32, 421-439.

Mongeon, P., \& Larivière, V. (2016). Costly collaborations: The impact of scientific fraud on co-authors' careers. Journal of the Association for Information Science \& Technology, 67, 535-542.

New York State Office of the Attorney General. (2010). The New York State Attorney General Andrew M. Cuomo announces charges against former UB researcher for hiring actors to testify during misconduct hearing and attempting to siphon \$4 million in taxpayer funds [Press Release]. Retrieved from https:// ag.ny.gov/press-release/2010/new-york-state-attorney-general-andrew-m-cuomo-announces-chargesagainst-former

Powers, M. B., Vedel, E., \& Emmelkamp, P. (2008). Behavioral couples therapy (BCT) for alcohol and drug use disorders: A meta-analysis. Clinical Psychology Review, 28, 952-962.

Sheehan, J. G. (2005). Fraud, conflict of interest, and other enforcement issues in clinical research. Cleveland Clinic Journal of Medicine, 74, S63-S67.

Stapel, D. A., \& Lindenberg, S. (2011). Coping with chaos: How disordered contexts promote stereotyping and discrimination. Science, 332, 251-253.

Titus, S. L., Wells, J. A., \& Rhoades, L. J. (2008). Repairing research integrity. Nature, 453, 980-982.

Tomsich, E.A., Tunstall, A.M., \& Gover, A.R. (2015). Couples counseling and domestic violence. The Encyclopedia of Crime \& Punishment. Retrieved from https://doi.org/10.1002/9781118519639.wbecp x 137

Wakefield, A. J., Murch, S. H., Anthony, A., Linnell, J., Casson, D. M., Malik, M., Berelowittz, M., Dhillon, A. P., Thompson, M. A., Harvey, P., Valentine, A., Davies, S. E., \& Walker-Smith, J. A. (1998). Ileallymphoid-nodular hyperplasia, non-specific colitis, and pervasive developmental disorder in children. Lancet, 351, 637-641.

Warner, G. (2010). Researcher accused of fraud is found dead. Retrieved from https://buffalonews.com/ news/researcher-accused-of-fraud-is-found-dead/article_814f56aa-dcff-5056-8f37-9368c47d5849.html

Wray, T. B., Grin, B., Dorfman, L., Glynn, T. R., Kahler, C. W., Marshall, B. D., ... Operario, D. (2016). Systematic review of interventions to reduce problematic alcohol use in men who have sex with men. Drug and Alcohol Review, 35, 148-157

Publisher's Note Springer Nature remains neutral with regard to jurisdictional claims in published maps and institutional affiliations. 